\title{
Acidosis tubular renal asociada a intoxicación por trimetoprim-sulfametoxazol
}

\author{
Renal tubular acidosis associated with \\ trimethoprim-sulfamethoxazole poisoning
}

\author{
Daniela Perdomo-Quintero, Camilo Ernesto Gómez-Barrios, Adriana Zamora-Suárez, \\ Juan Camilo Cifuentes-González • Neiva (Colombia)
}

DOI: https://doi.org/10.36104/amc.2020.1869

\section{Resumen}

La combinación de trimetoprim-sulfametoxazol (TMP-SMZ o cotrimoxazol) tiene efecto bactericida sobre cocos gram positivos y bacilos gram negativos, con uso clínico en infecciones de piel y tejidos blandos, del tracto respiratorio y urinario, además con relevancia en la profilaxis y tratamiento de infecciones oportunistas en pacientes inmunosupresos.

Su uso en pacientes inmunocompetentes a dosis establecidas es seguro, con una baja tasa de eventos adversos. Sin embargo, en población con inmunosupresión: virus de inmunodeficiencia humana (VIH), trasplante, o usuarios de esteroides; los efectos adversos (EA) por este medicamento alcanzan $83 \%$ y por administración parenteral en dosis elevadas se ha documentado acidosis láctica secundaria al vehículo polietilenglicol. Presentamos dos casos de pacientes que ingirieron TMP-SMZ en sobredosis, desarrollando acidosis tubular renal (ATR) tipo 2, la cual no se ha descrito en este medicamento y cuya hiperlactatemia no es explicable por el excipiente polietilenglicol debido a que el consumo fue oral. (Acta Med Colomb 2020; 45. DOI: https:// doi.org/10.36104/amc.2020.1869).

Palabras clave: trimetoprim y sulfametoxazol, intoxicación, acidosis tubular renal, hiponatremia.

\section{Abstract}

The combination of trimethoprim-sulfamethoxazole (TMP-SMZ or co-trimoxazole) has a bactericidal effect on gram-positive cocci and gram-negative bacilli. It is used clinically for skin and soft tissue, respiratory and urinary tract infections, and is also relevant for prophylaxis and treatment of opportunistic infections in immunosuppressed patients.

Its use at established doses in immunocompetent patients is safe, with a low rate of adverse events. However, in immunosuppressed individuals (human immunodeficiency virus [HIV], transplants, or steroid users), the adverse effects (AEs) of this medication reach $83 \%$; and, when administered parenterally at high doses, lactic acidosis has been documented secondary to the polyethylene glycol vehicle. We present two cases of patients who ingested an overdose of TMP-SMZ and developed type 2 renal tubular acidosis (RTA), which has not been described with this medication, and whose hyperlactatemia is not explained by the polyethylene glycol excipient, as it was taken orally. (Acta Med Colomb 2020; 45. DOI: https://doi.org/10.36104/amc.2020.1869).

Key words: trimethoprim and sulfamethoxazole, poisoning, renal tubular acidosis, hyponatremia.
Dra. Daniela Perdomo-Quintero: Médica General Universidad Surcolombiana; Dr. Camilo Ernesto Gómez-Barrios: Médico General Universidad Surcolombiana; Dra. Adriana ZamoraSuárez: Especialista en Toxicología Clínica, Hospital Universitario Hernando Moncaleano Perdomo, Docente Universidad Surcolombiana; Dr. Juan Camilo Cifuentes-González: Especialista en Medicina Interna, Hospital Universitario Hernando Moncaleano Perdomo, Docente Universidad Surcolombiana. Neiva (Colombia). Correspondencia: Dr. Juan Camilo Cifuentes González. Neiva (Colombia).

E-mail: chifu8600@gmail.com

Recibido: 09/V/2020 Aceptado: 30/VI/2020

\section{Introducción}

El trimetoprim-sulfametoxazol (TMP-SMZ o cotrimoxazol) es un antimicrobiano utilizado en el tratamiento de infecciones bacterianas y como profilaxis de infecciones oportunistas por Pneumocystis jiroveci en pacientes inmu- nocomprometidos. En la actualidad, se prescriben más de ocho millones anualmente (1).

$\mathrm{Su}$ uso en pacientes inmunocompetentes a dosis establecidas es seguro, con una baja tasa de eventos adversos (EA) aproximadamente $8 \%$ (2); sin embargo, en la pobla- 
ción inmunosupresa los EA pueden alcanzar alrededor de $83 \%$. En nuestro país, las intoxicaciones por medicamentos ocupan el segundo lugar con 20.9\% para un total de 14179 casos (3), dentro de este grupo los antibióticos cobran gran importancia al ser de frecuente prescripción en la práctica clínica. A continuación presentamos dos casos de pacientes que ingirieron de manera intencional dosis elevadas de TMP-SMZ e ingresaron al servicio de urgencias, su cuadro clínico y manejo médico establecido.

\section{Caso 1}

Mujer de 17 años sin antecedentes patológicos, quien ingresó al servicio de urgencias por cuadro clínico de cuatro horas de evolución consistente en ingesta auto infligida de 55 tabletas de TMP-SMZ, con posterior emesis, estupor, un episodio convulsivo tónicoclónico generalizado e hipotensión arterial; posteriormente entró en falla ventilatoria con requerimiento de intubación orotraqueal y Unidad de Cuidados Intensivos (UCI). Los paraclínicos iniciales, evidenciaron una marcada acidosis metabólica con hiperlactatemia (Tabla 1). Se instauró tratamiento con medidas de soporte general y bicarbonato de sodio IV. Posteriormente presentó estados de hiponatremia moderada e hipoglicemia. La paciente tuvo adecuada evolución clínica logrando extubación exitosa a los dos días de estancia hospitalaria. Sin embargo, persistió con acidosis metabólica que fue corregida sólo hasta el quinto día y fue dada de alta con seguimiento ambulatorio.

\section{Caso 2}

Hombre de 27 años, portador de VIH, con historia de infecciones oportunistas, quien ingresó al servicio de urgencias por cuadro clínico de episodios eméticos y somnolencia. Se tomaron paraclínicos iniciales que evidenciaron acidosis metabólica compensada con alcalosis respiratoria e hiperlactatemia. Posteriormente, presentó tres episodios de convulsiones tónicoclónicas generalizadas con supraversión de la mirada, sin relajación de esfínteres que fueron controladas con Midazolam IV. Esto sumado a episodios de agitación psicomotora plantearon la necesidad de descartar neuroinfección, por lo cual se decidió realizar tomografía computarizada y punción lumbar. También se realizó test de orina para descartar consumo de sustancias de abuso que fue reportado como negativo y los gases arteriales de control persistieron con acidosis metabólica (Tabla 1). Desde su ingreso fue tratado con medidas de soporte general por la sospecha de sobredosis con TMP-SMZ que se confirmó por el propio paciente al momento de recuperar estado de conciencia, indicando una ingesta total de 40 tabletas. Se corrige el estado ácido base al día cuarto de estancia hospitalaria y es dado de alta al quinto día.

\section{Discusión}

El TMP-SMZ es un antimicrobiano frecuentemente prescrito a nivel mundial. Aunque este fármaco en general es bien tolerado, su uso por más de cuarenta años ha permitido identificar algunos de los EA tanto en reacción adversa como en sobredosis. Se ha observado reacciones dermatológicas que van desde leves, como rash hasta severas como necrolisis epidérmica tóxica, siendo los pacientes con VIH los más afectados (1). Cuando se utiliza por largos periodos de tiempo, se ha asociado a alteraciones hematológicas como eosinofilia, anemia, trombocitopenia, agranulocitosis y aplasia medular (5). También se ha documentado casos de elevación de nitrogenados de forma transitoria o falla renal permanente, puede producir hiponatremia e hipercalemia, por lo cual se debe evitar utilizar junto a inhibidores de la enzima convertidora de angiotensina y espironolactona. Otros efectos más raros incluyen la hepatotoxicidad, vértigo y neumonitis (6).

En los anteriores casos clínicos observamos la presencia de acidosis metabólica con brecha aniónica normal o hiperclorémica, lo cual excluye causas como acidosis láctica, tóxicos como alcoholes o salicilatos, sepsis, insuficiencia renal o insuficiencia respiratoria, al mismo tiempo una de nuestras pacientes no tenia antecedentes patológicos, mientras que el otro, a pesar de tener como antecedente de importancia HIV, no cursaba con procesos infecciosos o alteraciones gastrointestinales que justificaran una pérdida de bicarbonato, por lo que la única posibilidad que restaba era una causa renal como la acidosis tubular renal (ATR).

Se ha reportado la inducción de acidosis láctica con dosis elevadas de presentaciones parenterales que contienen como vehículo polietilenglicol. Sin embargo, en nuestros pacientes no es posible considerar esta etiología, debido a que consumieron la presentación oral que carece de este excipiente (7).

Aunque en la literatura se describe la ATR tipo 4 como la más frecuente secundaria a TMP-SMZ, nuestros pacientes no presentaban hipercalemia por lo cual no se consideró. Por otro lado tenían un $\mathrm{pH}$ en orina inferior a 5.5, por lo que nos llevó a concluir que correspondían a una ATR tipo 2 , en donde hay incapacidad para reabsorber el bicarbonato a nivel del túbulo proximal, a diferencia de la ATR tipo 1 donde existe una incapacidad de los segmentos tubulares distales para acidificar adecuadamente la orina, lo que conduce a una alcalinización de ésta en presencia de acidosis metabólica (8).

La ART tipo 2 suele observarse en la tubulopatía de Fanconi, o por efecto de algunos medicamentos inhibidores de la anhidrasa carbónica como la acetazolamida y el topiramato. Los casos clínicos que reportamos no presentaban estos antecedentes, pero se ha descrito que el sulfametoxazol inhibe la anhidrasa carbónica, con los efectos ya explicados para el bicarbonato (9).

No existen estrategias de manejo basadas en evidencia o universalmente aceptadas para tratar la ATR secundaria a TMP-SMX. En la literatura se observa que con la suspensión del medicamento y medidas de soporte general que puede incluir en algunos casos, la alcalinización del medio con bicarbonato, los pacientes evolucionan adecuadamente (10). 
Tabla 1. Datos generales y paraclínicos de los pacientes.

\begin{tabular}{|c|c|c|c|c|c|c|c|}
\hline & Unidad & Paciente 1 & & & Paciente 2 & & \\
\hline Edad & años & 17 & & & 27 & & \\
\hline Sexo & & Femenino & & & Masculino & & \\
\hline Peso & $\mathrm{Kg}$ & 64 & & & 60 & & \\
\hline Comorbilidades & & Ninguna & & & VIH & & \\
\hline $\begin{array}{l}\text { Tmp-smz } \\
\text { No tabletas }\end{array}$ & $400 / 80 \mathrm{mg}$ & 55 & & & 40 & & \\
\hline Cocaína & & negativo & & & negativo & & \\
\hline Cannabinoides & & negativo & & & negativo & & \\
\hline Opioides & & negativo & & & negativo & & \\
\hline Metadona & & negativo & & & negativo & & \\
\hline Anfetaminas & & negativo & & & negativo & & \\
\hline Fenilciclidina & & negativo & & & negativo & & \\
\hline Benzodiacepinas & & negativo & & & negativo & & \\
\hline Barbitúricos & & negativo & & & negativo & & \\
\hline Meta-anfetaminas & & negativo & & & negativo & & \\
\hline A.tricíclicos & & negativo & & & negativo & & \\
\hline TAC de cráneo & & Normal & & & $\begin{array}{l}\text { leve atrofia de la corteza frontal } \\
\text { sin lesiones aparentes de neu- } \\
\text { roinfección oportunista }\end{array}$ & & \\
\hline \multirow[t]{2}{*}{ Punción lumbar } & & - & & & Dentro parámetros normales & & \\
\hline & & Dia 1 & Dìa 2 & Día 3 & Día 1 & Día 2 & Día 3 \\
\hline Glicemia & $\mathrm{mg} / \mathrm{dL}$ & 188 & 67 & 112 & 127 & 69 & 91 \\
\hline BUN & $\mathrm{mg} / \mathrm{dL}$ & 4.9 & 6.6 & & 12 & 15 & 14 \\
\hline Creatinina & $\mathrm{mg} / \mathrm{dL}$ & 0.64 & 0.68 & & 1,05 & 1.3 & 1.1 \\
\hline AST & $\mathrm{UI} / \mathrm{L}$ & 21.4 & 28 & & 30 & 49 & 40 \\
\hline ALT & $\mathrm{UI} / \mathrm{L}$ & 39 & 37 & & 35 & 34 & 35 \\
\hline FA & $\mathrm{U} / \mathrm{L}$ & 82 & & & 87 & & \\
\hline $\mathrm{Hb}$ & $\mathrm{g} / \mathrm{dL}$ & 13.5 & 11.4 & & 14 & & \\
\hline Hto & $\%$ & 41.3 & 36.5 & & 42.4 & & \\
\hline $\mathrm{VCM}$ & $\mathrm{fL}$ & 79 & 82.9 & & 83 & & \\
\hline $\mathrm{Na}$ & $\mathrm{mmol} / \mathrm{L}$ & 135 & 130 & 134 & 138 & 140 & 142 \\
\hline $\mathrm{Ca} \mathrm{i}$ & $\mathrm{mmol} / \mathrm{L}$ & 1.09 & 1.1 & 1.2 & 1.1 & 1.2 & 1.0 \\
\hline $\mathrm{k}$ & $\mathrm{mmol} / \mathrm{L}$ & 4.5 & 3.94 & 3.7 & 4.2 & 3,8 & 4 \\
\hline $\mathrm{Cl}$ & $\mathrm{mEq} / \mathrm{L}$ & 117 & 108 & 105 & 119 & 111 & 106 \\
\hline $\mathrm{pH}$ & & 7.09 & 7.27 & 7.39 & 6.92 & 7.21 & 7.39 \\
\hline $\mathrm{PCO}_{2}$ & $\mathrm{mmHg}$ & 24 & 32 & 35.9 & 21 & 29 & 36 \\
\hline $\mathrm{PO}_{2}$ & $\mathrm{mmHg}$ & 99 & 156 & 90.8 & 93 & 95 & 97 \\
\hline $\mathrm{HCO}_{3}$ & $\mathrm{mmol} / \mathrm{L}$ & 7.3 & 16.3 & 21.4 & 7.2 & 17.9 & 24.2 \\
\hline Lactato & $\mathrm{mmol} / \mathrm{L}$ & 11.2 & 1.4 & 0.91 & 15 & 9 & 1.1 \\
\hline $\mathrm{BE}$ & $\mathrm{mmol} / \mathrm{L}$ & & -11.1 & -3.5 & & & \\
\hline AG & $\mathrm{mEq} / \mathrm{L}$ & 10.7 & & & 11,8 & 11.1 & 11.8 \\
\hline $\mathrm{pH}$ urinario & & 4.9 & & & 5.1 & & \\
\hline
\end{tabular}


Finalmente la rápida resolución del cuadro clínico en ambos casos con las medidas terapéuticas empleadas, apoya nuestra hipótesis y el tratamiento instaurado.

La paciente del primer caso presentó discreta hiponatremia que se resolvió al tercer día de hospitalización. Al comparar este hallazgo con la literatura, encontramos que es poco frecuente, pero se explicaría por la acción que tiene el TMP a nivel del túbulo distal; ya que es una base débil heterocíclica que posee una estructura similar a los diuréticos ahorradores de potasio como el amiloride y triamtereno, bloqueando la reabsorción de sodio a nivel del canal epitelial de sodio en la región distal de la nefrona (11). En nuestros pacientes no se evidenció hipercalemia que es una alteración que se puede observar en $44-70 \%$ en quienes reciben TMP-SMZ. Sin embargo, es más probable que se reporte en aquellos que presentan $\mathrm{VIH}$ y han requerido uso prolongado del antibiótico ya sea como tratamiento o como profilaxis (12), situación que no se presentó en el paciente con VIH, ya que no había consumido el medicamento en los últimos seis meses antes de la sobredosis por la cual consultó.

Finalmente, la hipoglicemia es ocasionada por el sulfametoxazol que puede aumentar la liberación de insulina por parte del páncreas, especialmente en casos de sobredosis como los que reportamos sumado a insuficiencia renal (13). Esto probablemente por la semejanza estructural del sulfametoxazol a las sulfonilureas.

Como podemos observar los EA relacionados con el uso de TMP SMX si bien, se resuelven luego de suspender la terapia o la disminución de la dosis, son complicaciones que deben ser identificadas tempranamente para disminuir la morbilidad y la estancia hospitalaria. Así mismo se requiere investigar aún más sobre los mecanismos de acción implicados para una prescripción adecuada, especialmente en individuos susceptibles como los pacientes inmunosupresos (VIH- trasplante y usuarios de corticosteroides).

\section{Referencias}

1. Yang, J. J., Huang, C. H., Liu, C. E., Tang, H. J., Yang, C. J., Lee, Y. C., Hung, C. C. Multicenter study of trimethoprim/sulfamethoxazole-related hepatotoxicity: Incidence and associated factors among HIV-infected patients treated for Pneumocystis jirovecii pneumonia. PLoS ONE, 9(9). DOI: 10.1371/journal. pone. 0106141

2. Vander Ven AJ, Koopmans PP, Vree TB, vander Meer JW. Adversereactions toco-trimoxazole in HIV infection. Lancet. 1991;338:431-433. DOI: 10.1016/0140-6736(91)91046-w

3. Arun Mattappalil and KariA. Mergenhagen. Neurotoxicity with Antimicrobials in the Elderly: A Review Clin Ther. 2014;36:1489-1511 DOI: 10.1016/j. clinthera.2014.09.020

4. INS. Semana epidemiológica 47. Available at: https://www.minsalud.gov.co/ Paginas/Colombia-se-acerca-a-la- (Accessed: 1 February 2020).

5. Walker, S., Norwood, J., Thornton, C., \& Schaberg, D. Trimethoprimsulfamethoxazole associated rhabdomyolysis in a patient with AIDS: Case report and review of the literature. American Journal of the Medical Sciences, 331(6), 339-341 DOI: 10.1097/00000441-200606000-00011

6. INS. Semana epidemiológica 45. Available at: https://www.minsalud.gov.co/ Paginas/Colombia-se-acerca-a-la- (Accessed: 1 February 2020).

7. Bulathsinghala, M., Keefer, K. and Van de Louw, A. (2016) 'Trimethoprim/ Sulfamethoxazole-Induced Severe Lactic Acidosis', Medicine. Lippincott Williams and Wilkins, 95(17), p. e3478. DOI: 10.1097/MD.0000000000003478

8. Karet FE. Mechanisms in hyperkalemic renal tubular acidosis. J Am Soc Nephrol 2009;20(2):251-4. DOI: 10.1681/ASN.2008020166

9. Kamel KS, Briceno LF, Sanchez MI, et al. A new classification for renal defects in net acid excretion. Am J Kidney Dis 1997; 29(1): 136-46. DOI: 10.1016/ s0272-6386(97)90021-4

10.Purkerson JM, Schwartz GJ: The role of carbonic anhydrases in renal physiology . Kidney Int.2007, 71:103-115. DOI: 10.1038/sj.ki.5002020

11. Bulathsinghala M, Keefer K, Van de Louw A. Trimethoprim/SulfamethoxazoleInduced Severe Lactic Acidosis: A Case Report and Review of the Literature. Medicine (Baltimore). 2016; 95(17): e3478. doi:10.1097/MD.0000000000003478

12. Yorgin PD, Theodorou AA, Al-Uzri A, et al. Propylene glycolinduced proximal renal tubular cell injury. Am J Kidney Dis. 1997; 30: 134-139. DOI: 10.1016/ s0272-6386(97)90577-1

13. Mori H., Kuroda Y., Imamura S., et al: Hyponatremia and/or hyperkalemia in patients treated with the standard dose of trimethoprim-sulfamethoxazole. Intern Med 2003; 42: pp. 665-669. DOI: 10.2169/internalmedicine.42.665 